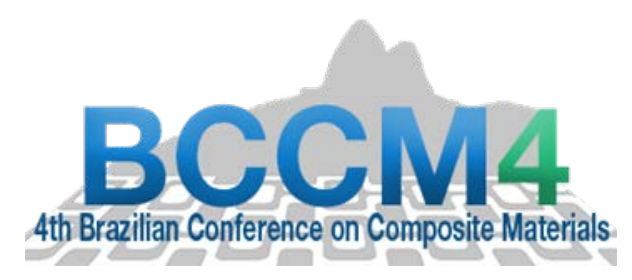

\title{
NUMERICAL MODELLING OF HELICAL CABLES USING BEAM ELEMENTS
}

\author{
$\underline{\text { Eduardo A. W. de Menezes }}^{(1)}$, Rogério J. Marczak ${ }^{(1)}$ and Sandro C. Amico ${ }^{(2)}$ \\ (1) Department of Mechanical Engineering, Federal University of Rio do Sul, Brazil \\ (2) Department of Materials Engineering, Federal University of Rio do Sul, Brazil
}

https://doi.org/10.21452/bccm4.2018.16.04

\begin{abstract}
Due to the complex geometry of helical cables and wire ropes, the available analytical models have a series of simplifications, assumptions and a limited capacity in reproducing their mechanical behavior, and 3D numerical models are often costly and time-consuming due to interwire contact. Both methods are not viable when dealing with long cables, as in cable-stayed bridges and offshore platforms applications, where they can reach more than $1500 \mathrm{~m}$ (ultra-deep waters). The purpose of this work is to incorporate a new $1 \mathrm{D}$ beam element in a commercial finite element (FE) software. A 3D FE model, previously verified by experiments, was used to calibrate the $1 \mathrm{D}$ element, which carries the information provided by the 3D model in its stiffness matrix. The result is a 2-node beam element with six degrees of freedom per node which is able to simulate long cables, combining both the practical implementation of an analytical model with the accuracy of a 3D FE model. The adjusted beam model fitted the 3D model with a coefficient of determination $\left(\mathrm{R}^{2}\right)$ above 0.90 .
\end{abstract}

Keywords: Cables, Finite element modelling, beam element.

\section{INTRODUCTION}

A cable or a wire rope is a structural element made of a layup of strands and wires or fibers helically wrapped around a central straight core [1]. Their applications include prestressing of concrete, stays for guyed masts [1], deployable structures for satellite transportations [2] bridging applications [3] and mooring of offshore platforms [4]. In all these applications, carbon-fiber reinforced polymer (CFRP) cables are replacing traditional materials due to their high specific strength and stiffness compared to metallic and synthetic fiber cables [5], outstanding fatigue behavior [3], no-magnetism, corrosion resistance [2] and low thermal expansion, being also superior to steel in critical mechanical aspects as creep and relaxation [6]. Their disadvantages, however, include lack of ductility, high cost, and difficulties related to connections [6]. 
The first mathematical models created to predict its mechanical behavior considered the cable as a set of bars, neglecting torsion and bending stiffness, such as the model proposed by Hruska [7]. In the 70's and 80's different authors applied Love's rod theory [8] to model cables, as in Costello's model for the tensile and bending behavior of single and multi-layered cables [9]. These models, however, made many assumptions, including linear elastic infinitesimal elasticity, isotropic material, neglection of contact forces, and limited applicability to a few boundary conditions [10]. Ghoereishi [1] compared classical analytical models and a finite element (FE) model and showed that for low helix angles (low pitch cables), analytical and numerical models diverge substantially.

More recently, different models were reported to tackle some of these assumptions. Elata [11] proposed a model to accurately capture stress distribution along the wires of multi-layered cables, which has been reported to be poorly predicted by most analytical models like Costello's [12], while Argatov [13] attempted to model interwire friction and Crossley [14] created a model for a transversally isotropic material. Nevertheless, none of them could overcome all hypotheses, which is desirable for a trustworthy model for structural cables.

Regarding numerical solutions, a good correlation can be achieved between FE models and experimental tests (tensile or bending), even for helical composite cables [15]. However, it has been shown that the required computational time to simulate helical cables with 3D hexahedral elements greatly increases as a function of the cable length. For dynamic simulations of an inservice cable in offshore platforms, for instance, it is recommended to simulate its full length, whose value for ultra-deep water is greater than $1500 \mathrm{~m}$.

This work proposes a methodology to model a helical cable using Euler-Bernoulli beam elements in order to combine the accuracy of 3D FE models with the quickness of analytical models. The process consists in numerically evaluating the stiffness matrix of a transversally isotropic cable and then apply the least square method to compute coefficients that could adjust an Euler-Bernoulli stiffness matrix to the stiffness of the 3D model.

\section{METHODOLOGY}

\subsection{Beam element}

Beams are the most common type of structural components, widely used in civil and mechanical engineering. Since a 3D body is modeled as a 1D body, several assumptions and approximations to the underlying physics are made [16]. Classical beam theory of Euler-Bernoulli, which is eligible for slender beams [17], involves the following assumptions [18]: (i) one dimension is considerably larger than the other two; (ii) plane cross-sections perpendicular to the axis of the beam remain plane and perpendicular to the axis after deformation; (iii) shear deformation is neglected; (iv) deformations are infinitesimal; (v) the Poisson effect is neglected; (vi) symmetric cross-sectional area, with the neutral axis coincident with the centroid; (vii) linear-elastic material. Since the focus here is on long composite cables, usually CFRP (with ultimate strain ca. 2\%), hypotheses (i-vi) are valid, whereas hypothesis (vii) must be further investigated.

In order to evaluate the beam element stiffness matrix, a two-node element is considered, with six degrees of freedom (DOF) per node (namely, axial displacement, transverse displacement in $y$-direction, transverse displacement in $z$-direction, and rotation about $x, y$ and $z$-axis) depicted in Figure 1, where $u$ are displacements and $\theta$ rotations, with subscripts indicating node number and direction, respectively.

For DOF 1 (axial displacement), considering a beam with length $L$, constant cross-section area $A$ and Young's modulus $E$, the stiffness is as follows: 


$$
K_{i j}=E A \int_{x_{1}}^{x_{2}} \frac{d \psi_{i}(x)}{d x} \cdot \frac{d \psi_{j}(x)}{d x} d x
$$

where $i$ and $j$ are stiffness coefficients (for indices 1 and 7), and $\psi$ represents the interpolation functions:

$$
\begin{aligned}
& \psi_{1}=1-\frac{x_{2}-x_{1}}{L}, \\
& \psi_{7}=\frac{x_{2}-x_{1}}{L}
\end{aligned}
$$

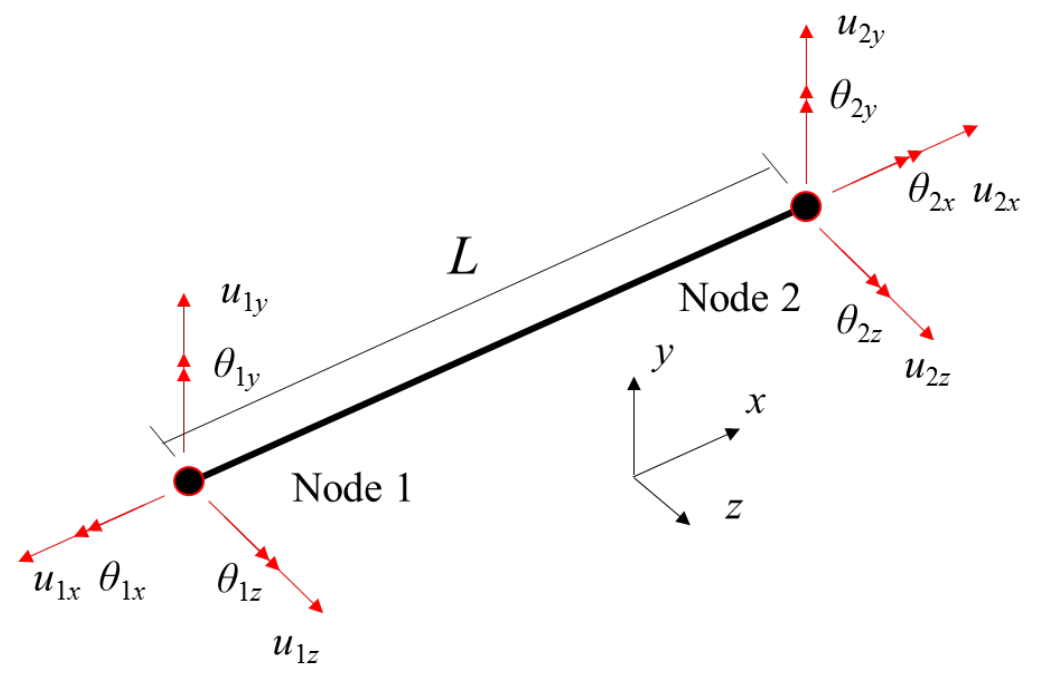

Figure 1 - Illustration of the beam element and coordinate system.

Regarding DOFs 2 and 5 (deflection in $y$ and rotation around the $z$-axis, respectively), Hermite interpolation functions were applied, and the stiffness is given by:

$$
K_{i j}=E I_{z} \int_{x_{1}}^{x_{2}} \frac{d \psi_{i}^{2}(x)}{d x^{2}} \cdot \frac{d \psi_{j}^{2}(x)}{d x^{2}} d x
$$

for

$$
\begin{aligned}
& \psi_{2}=1-3\left(\frac{x_{2}-x_{1}}{L}\right)^{2}+2\left(\frac{x_{2}-x_{1}}{L}\right)^{3} \\
& \psi_{6}=\left(x_{2}-x_{1}\right)\left(1-\frac{x_{2}-x_{1}}{L}\right)^{2} \\
& \psi_{8}=3\left(\frac{x_{2}-x_{1}}{L}\right)^{2}-2\left(\frac{x_{2}-x_{1}}{L}\right)^{3} \\
& \psi_{12}=\left(x_{2}-x_{1}\right)\left[\left(\frac{x_{2}-x_{1}}{L}\right)^{2}-\frac{x_{2}-x_{1}}{L}\right]
\end{aligned}
$$


where $i$ and $j$ are indices 2, 6, 8 and 12 and $I_{z}$ is the moment of inertia about $z$-axis. Analogously, DOFs 3 and 6 (deflection in $z$ and rotation around the $y$-axis, respectively) are obtained with the same interpolation functions of Equation (4), but changing $I_{z}$ for $I_{y}$ in Equation (3). DOF 4 (torsion around the $x$-axis) is achieved by substituting $E$ for $G$ (shear modulus) and $A$ for $J$ (polar moment of inertia) in Equation (1), through the interpolation functions of Equation (2). The complete stiffness matrix is obtained as:

$K=\left[\begin{array}{cccccccccccc}\frac{E A}{L} & 0 & 0 & 0 & 0 & 0 & -\frac{E A}{L} & 0 & 0 & 0 & 0 & 0 \\ 0 & \frac{12 E I_{z}}{L^{3}} & 0 & 0 & 0 & \frac{6 E I_{z}}{L^{2}} & 0 & -\frac{12 E I_{z}}{L^{3}} & 0 & 0 & 0 & \frac{6 E I_{z}}{L^{2}} \\ 0 & 0 & \frac{12 E I_{y}}{L^{3}} & 0 & -\frac{6 E I_{y}}{L^{2}} & 0 & 0 & 0 & -\frac{12 E I_{y}}{L^{3}} & 0 & -\frac{6 E I_{y}}{L^{2}} & 0 \\ 0 & 0 & 0 & \frac{G J}{L} & 0 & 0 & 0 & 0 & 0 & -\frac{G J}{L} & 0 & 0 \\ 0 & 0 & -\frac{6 E I_{y}}{L^{2}} & 0 & \frac{4 E I_{y}}{L} & 0 & 0 & 0 & \frac{6 E I_{y}}{L^{2}} & 0 & \frac{2 E I_{y}}{L} & 0 \\ 0 & \frac{6 E I_{z}}{L^{2}} & 0 & 0 & 0 & \frac{4 E I_{z}}{L} & 0 & -\frac{6 E I_{z}}{L^{2}} & 0 & 0 & 0 & \frac{2 E I_{z}}{L} \\ -\frac{E A}{L} & 0 & 0 & 0 & 0 & 0 & \frac{E A}{L} & 0 & 0 & 0 & 0 & 0 \\ 0 & -\frac{12 E I_{z}}{L^{3}} & 0 & 0 & 0 & -\frac{6 E I_{z}}{L^{2}} & 0 & \frac{12 E I_{z}}{L^{3}} & 0 & 0 & 0 & -\frac{6 E I_{z}}{L^{2}} \\ 0 & 0 & -\frac{12 E I_{y}}{L^{3}} & 0 & \frac{6 E I_{y}}{L^{2}} & 0 & 0 & 0 & \frac{12 E I_{y}}{L^{3}} & 0 & \frac{6 E I_{y}}{L^{2}} & 0 \\ 0 & 0 & 0 & -\frac{G J}{L} & 0 & 0 & 0 & 0 & 0 & \frac{G J}{L} & 0 & 0 \\ 0 & 0 & -\frac{6 E I_{y}}{L^{2}} & 0 & \frac{2 E I_{y}}{L} & 0 & 0 & 0 & \frac{6 E I_{y}}{L^{2}} & 0 & \frac{4 E I_{y}}{L} & 0 \\ 0 & \frac{6 E I_{z}}{L^{2}} & 0 & 0 & 0 & \frac{2 E I_{z}}{L} & 0 & -\frac{6 E I_{z}}{L^{2}} & 0 & 0 & 0 & \frac{4 E I_{z}}{L}\end{array}\right]$

\subsection{D FE Model}

The cable was modelled in the FE commercial platform Abaqus using the C3D8R element (hexahedral linear eight-node with reduced integration) [19]. The geometry adopted, $1 \times 7$, is shown in Figure 2 (a). Hard and tangential contacts (penalty method) were inserted between external wires and between wires and core (Figure 2 (b)). Since the material is considered transversally isotropic, a local coordinate system was used to keep the wires' properties aligned in the correct orientation. 


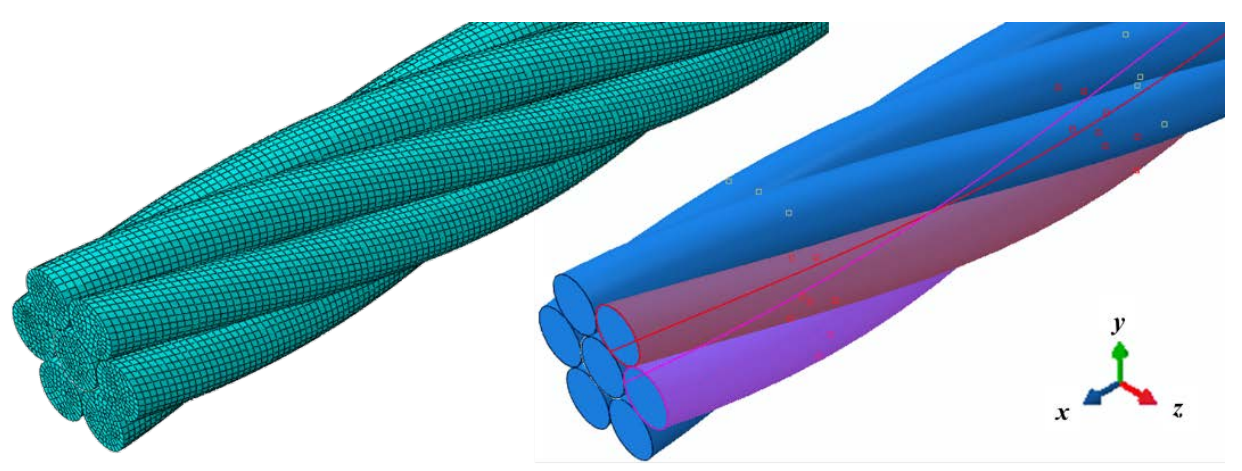

(a)

(b)

Figure 2 - (a) $1 \times 7$ cable meshed with the C3D8R elements and (b) one of the 12 contact pairs inserted in the model.

The procedure to obtain the stiffness components is illustrated in Figure 3 and consists in clamping the cable in the left end and allowing 1 DOF free in the right end. The stiffness is obtained by measuring the reaction forces or moments after applying the prescribed displacements or rotations. Since the $I_{y}$ and $I_{z}$ values are equal, DOFs 2 and 6 are equal to 3 and 5 , respectively. For the DOF 4, rotation was applied clockwise and anti-clockwise since for a helical cable torsion stiffness is expected to be higher when twisting it in a direction opposite to the wires initial orientation. The other terms of the beam stiffness matrix were omitted due to matrix symmetry and assumption of $I_{y}=I_{z}$.

(a)

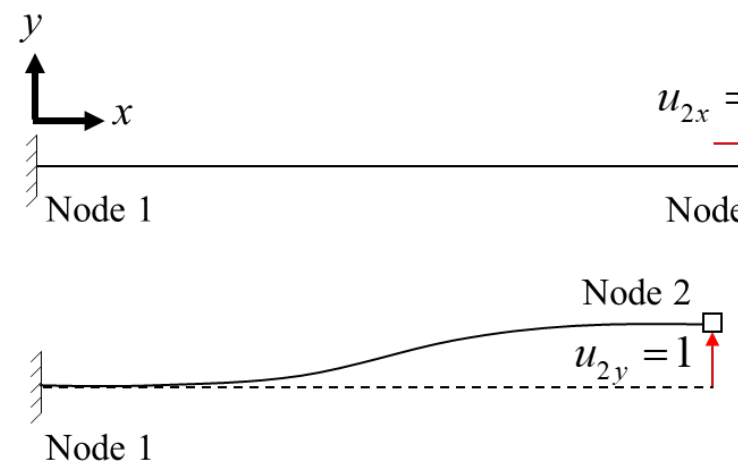

$$
K_{11}=K_{77}=-K_{17}=F_{1 x}=\frac{E A}{L}
$$

(b)

Node 1

$$
\begin{aligned}
& K_{22}=K_{88}=-K_{28}=F_{2 y}=\frac{12 E I}{L^{3}} \\
& K_{26}=K_{2,12}=-K_{68}=-K_{8,12}=M_{1 z}=\frac{6 E I}{L^{2}}
\end{aligned}
$$

(c)

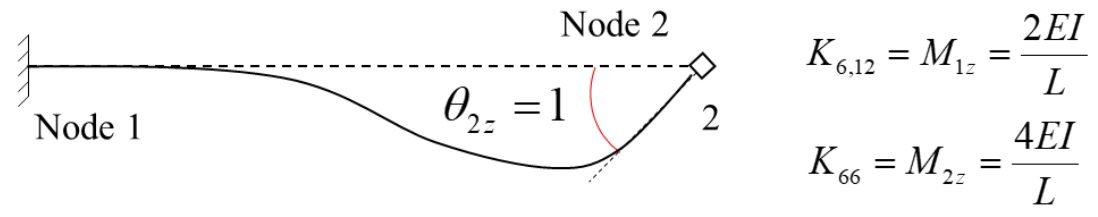

(d)

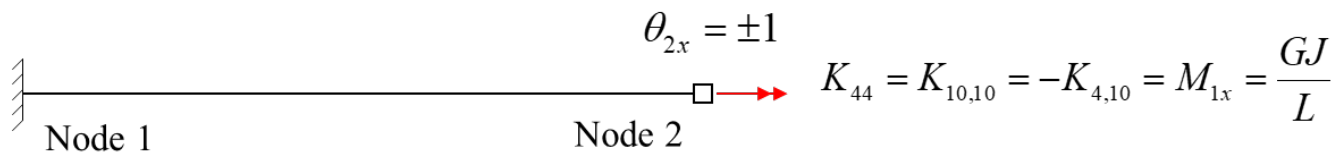

Figure 3 - Procedure adopted to obtain six terms of the beam stiffness matrix. 


\subsection{Regression procedure}

The factorial design allows the simultaneous consideration of many variables at different levels, as well as the interaction between them [20]. In order to define the required level for each variable, a sensitive analysis was performed involving all parameters that significantly impact the cable behavior. Considering the material as transversally isotropic, engineering constants $v_{12}=v_{13}, v_{23}$, $G_{23}, E_{2}=E_{3}$ and the friction coefficient $\mu$ play a small role in the cable behavior [15], therefore, the sensitivity analysis focused on wire diameter $D$, longitudinal Young's modulus $E_{1}$, in-plane shear modulus $G_{12}=G_{13}$, helix angle $\alpha$ and length $L$. The variables were individually studied.

After defining the factorial design, coefficients to adjust the cable stiffness to the beam stiffness matrix of Equation (5) were numerically evaluated through finite-differences method [21]. Different function families were tried to maximize the coefficient of determination $\left(\mathrm{R}^{2}\right)$.

\section{RESULTS AND DISCUSSION}

The values used as input in the 3D model to evaluate cable stiffness are reported in Table 1, while the ranges of the sensitivity analysis are reported in Table 2. Relative to shear modulus, its influence was only significant in DOF 4, and for a long cable, the difference in stiffness considering rotation applied clockwise and anti-clockwise was negligible. Its influence in DOF 4 (torsion term) was approximately linear $\left(\mathrm{R}^{2}>0.999\right)$.

Regarding the Young's modulus, its influence in the torsion stiffness was negligible and approximately linear for all the other stiffness matrix terms (average value of $\mathrm{R}^{2}$ above 0.98). Relative to length and diameter, linear behavior was observed in all DOFs. However, while the linearity in $L$ increases with cable length, the opposite occurred for the diameter. For the analysis of $\alpha$, a review in scientific and technical literature was carried out to identify the angle range for real cables, which was found to be within $62^{\circ}-82^{\circ}$. As the helix angle increases, all stiffness terms increase, but at distinct and non-linear rates.

Table 1 - Values adopted for the numerical model.

\begin{tabular}{c|c} 
Property & Value \\
\hline Geometry & $1 \times 7$ (6 wires+core $)$ \\
$E_{2}=E_{3}(\mathrm{MPa})$ & 180000 \\
$G_{23}(\mathrm{MPa})$ & 69231 \\
$v_{12}=v_{13}=v_{23}$ & 0.30 \\
$\mu$ & 0.65
\end{tabular}

Table 2 - Ranges of the analyzed variables.

\begin{tabular}{c|c|c} 
Parameter & Minimum & Maximum \\
\hline$E_{1}(\mathrm{MPa})$ & 90000 & 270000 \\
$G_{12}(\mathrm{MPa})$ & 34615 & 103846 \\
$D(\mathrm{~mm})$ & 2.12 & 3.67 \\
$L(\mathrm{~mm})$ & 50 & 150 \\
$\alpha\left(^{\circ}\right)$ & 62 & 82
\end{tabular}

Based on the aforementioned results, two levels were adopted for $E$, $G$, and $L$, three for $D$ and five for $\alpha$. Considering the four cases (a-d) mentioned in Figure 3, the total number of simulations 
was $(2 \times 2 \times 3 \times 5) \times 4=240$. These simulations were performed considering the ranges exposed in Table 2, except for the length, whose value was the largest possible within the convergence range, aiming to achieve a better fitting for the proposed application. The applied lengths and updated stiffness coefficients are reported in Table 3 , along with the $\mathrm{R}^{2}$ values, which were greater than 0.9 in all cases.

Considering that all terms in the stiffness matrix of the beam element were adjusted with relative good accuracy, this beam model is expected to satisfactory reproduce a $1 \times 7$ cable behavior under different load conditions, with an extremely low computational time in comparison to $3 \mathrm{D}$ models with contact between wires, since this feature is incorporated in the updated beam model.

Table 3 - Updated values of stiffness after the adjustment process, along with the respective coefficients of determination.

\begin{tabular}{c|c|c} 
Length Range & Adjusted Stiffness & $\mathrm{R}^{2}$ \\
\hline $100-150 \mathrm{~mm}$ & $K_{11}=-2.641 \frac{E A}{L}+3.598 \frac{E A}{L} \sin (\alpha)$ & 0.9994 \\
$100-200 \mathrm{~mm}$ & $K_{22}=3.041 \frac{12 E I}{L^{3}}-2.756 \frac{12 E I}{L^{3}} \sin (\alpha)-1.184 \frac{12 E I}{L^{3}} \cos (\alpha)$ & 0.9547 \\
$100-200 \mathrm{~mm}$ & $\begin{array}{c}K_{26}=2.756 \frac{6 E I}{L^{2}}-2.492 \frac{6 E I}{L^{2}} \sin (\alpha)-1.077 \frac{6 E I}{L^{2}} \cos (\alpha) \\
100-150 \mathrm{~mm}\end{array}$ & 0.9423 \\
$100-150 \mathrm{~mm}$ & $K_{66}=0.012 \frac{4 E I}{L}-0.015 \frac{4 E I}{L} \tan (\alpha)$ & 0.9117 \\
$100-200 \mathrm{~mm}$ & $K_{6,12}=-0.002 \frac{2 E I}{L}+0.020 \frac{2 E I}{L} \tan (\alpha)$ & 0.9006 \\
$K_{44}=-0.035 \frac{G J}{L}+0.060 \frac{G J}{L}$ & 0.9180
\end{tabular}

\section{CONCLUSIONS}

A methodology for the simulation of cables using 3D beam elements was presented. Higher coefficients of determination were obtained when comparing the beam stiffness with the stiffness of a 3D cable, indicating that the beam mechanical behavior will accurately simulate the behavior of the 3D cable. This model is able to simulate long cables much faster than 3D models. The use of trigonometric functions in the stiffness terms that account for the helix angle is justifiable since the cable is composed of one straight (core) and six helical wires.

The influence of the ratio between core and external wires diameter on the updated stiffness must be further investigated, as well as the application of this methodology for multi-layered cables. And the proposed methodology still needs to be validated to verify the implications of neglecting non-linearities and the coupling between tension and torsion.

\section{ACKNOWLEDGEMENTS}

The authors gratefully acknowledge the National Council for Scientific and Technological Development (CNPq). 
$4^{\text {th }}$ Brazilian Conference on Composite Materials. Rio de Janeiro, July $22^{\text {nd }}-25^{\text {th }}$, 2018

\section{REFERENCES}

[1] Ghoreishi, S. R., Messager, T., Cartraud, P., Davies, P., 'Validity and limitations of linear analytical models for steel wire strands under axial loading, using a 3D FE model', Int. J. of Mech. Sci.49 (2007) 1251-1261.

[2] Motoyama, H., Ohta, T., Ohno, T., Moriya, K. 'Dynamic Tensile Properties of CFRP Cables Subjected to High-speed Loads’, Mem of Fac of Eng. 62 (3) (2002) 113-127.

[3] Meier, U., 'Carbon Fiber Reinforced Polymer Cables: Why? Why Not?', Arab. J. Sci. Eng. 37 (2012) 399-411.

[4] Sparks, C., Zivanovic, I., Luyckx, J., Hudson, W., 'Carbon Fiber Composite Tendons for Deepwater Tension Leg Platforms', in 'Offshore Technology Conference', Houston, Texas, USA, May, 2003.

[5] Jackson, D. et. al., 'Carbon Fiber Composite Tendons for Deepwater Tension Leg Platforms', in 'Offshore Technology Conference', Houston, Texas, USA, May, 2005.

[6] Xie, X., Li, X., Shen, Y., 'Static and Dynamic Characteristics of a Long-Span Cable-Stayed Bridge with CFRP Cables', Materials 7 (2014) 4854-4877.

[7] Hruska, F. H., 'Calculation of stresses in wire ropes', Wire and Wire Prod., 26 (9) (1951) 766-767.

[8] Love, A. E. H., 'A Treatise on the Mathematical Theory of Elasticity', 4th Edn (Dover Publications, New York, 2011).

[9] Costello, G. A., 'Theory of Wire Rope’, 1st Edn (Springer-Verlag, New York, 1990).

[10]Cardou, A., Jolicoeur, C., 'Mechanical models of helical strands', Appl Mech. Rev., 50 (1) (1997) 1-14.

[11]Elata, D., Eshkenazy, R., Weiss, M. P., 'The mechanical behavior of a wire rope with an independent wire rope core', Int. J. of Sol. and Str., 41 (2004) 1157-1172.

[12]Menezes, E. A. W., et. al., 'Numerical and Experimental Analysis of the Tensile and Bending Behavior of CFRP Cables', Pol. and Pol. Comp. 25 (9) (2017) 643-650.

[13]Argatov, I., 'Response of a wire rope strand to axial and torsional loads: Asymptotic modelling of the effect of interwire contact deformations', Int. J. of Sol. and Str.,48 (2011) 1413-1423.

[14]Crossley, J. A., Spencer, A. J. M., England, A. H., 'Analytical solutions for bending and flexure of helically reinforced cylinders’, Int. J. of Sol. and Str., 40 (2003) 777-806.

[15]Menezes, E. A. W., Silva, L. V., Marczak, R. J., Amico, S. C., 'Numerical model updating applied to the simulation of carbon fiber-reinforced polymer cables under bending and tensile stress', J. of Strain Analysis, 52 (6) (2017) 356-364.

[16]Felippa, C. A., 'Introduction to Finite Element Methods', (University of Colorado, 2004).

[17]Lee, S. J., Park, K. S., 'Vibrations of Timoshenko beams with isogeometric approach', Applied Math. M., 37 (2013) 9174-9190.

[18]Han, S. M., Benaroya, M., Wei, T., 'Dynamics of transversely vibrating beams using four engineering theories', J. of Sound and Vib., 225 (5) (1999) 935-988.

[19]Abaqus 2016, SIMULIA Academic Research, Dassault Systèmes Americas Corp, Waltham, Massachusetts, USA, 2016.

[20]Berrios, M., Gutiérrez, M. C., Martín, M. A., Martín, A., Application of the factorial design of experiments to biodiesel production from lard', Fuel Pro. Tech., 90 (12) (2009) 1447-1451.

[21]MATLAB R. version 8.0.0.783 (R2012b), The MathWorks Inc., Natick, Massachusetts, USA, 2012. 